\title{
Prospects on searches for baryonic Dark Matter produced in $b$-hadron decays at $\mathrm{LHCb}$
}

\author{
Alexandre Brea Rodríguez ${ }^{1, \mathrm{a}}$, Veronika Chobanova ${ }^{1, \mathrm{~b}}$, Xabier Cid Vidal $^{1, \mathrm{c}}{ }_{\odot}$, Saúl López Soliño ${ }^{1, \mathrm{~d}}$, \\ Diego Martínez Santos ${ }^{1, \mathrm{e}}$, Titus Mombächer ${ }^{1, \mathrm{f}}$, Claire Prouvée ${ }^{1, \mathrm{~g}}$, Emilio Xosé Rodríguez Fernández ${ }^{1, \mathrm{~h}}$, \\ Carlos Vázquez Sierra ${ }^{2, i}{ }_{i}$ \\ ${ }^{1}$ Instituto Galego de Física de Altas Enerxías (IGFAE), Universidade de Santiago de Compostela, 15782 Santiago de Compostela, Spain \\ ${ }^{2}$ European Organization for Nuclear Research (CERN), Geneva, Switzerland
}

Received: 23 June 2021 / Accepted: 19 October 2021 / Published online: 2 November 2021

(c) The Author(s) 2021

\begin{abstract}
A model that can simultaneously explain Dark Matter relic density and the apparent matter anti-matter imbalance of the universe has been recently proposed. The model requires $b$-hadron branching fractions to Dark Matter at the per mille level. The $b$-hadrons decay to a dark sector baryon, $\psi_{\text {DS }}$, which has a mass in the region $940 \mathrm{MeV} / c^{2} \leq$ $m_{\psi_{\text {DS }}} \leq 4430 \mathrm{MeV} / c^{2}$. In this paper, we discuss the sensitivity of the $\mathrm{LHCb}$ experiment to search for this dark baryon, covering different types of topology and giving prospects for Runs 3 and 4 of the LHC, as well as for the proposed Upgrade II. We show that the $\mathrm{LHCb}$ experiment can cover the entire mass range of the hypothetical dark baryon.
\end{abstract}

\section{Introduction}

Despite the long standing success of the Standard Model (SM) of particle physics, there is experimental evidence for New Physics that is not explained by the SM. The most tantalising signs for New Physics are the apparent existence of Dark Matter and the matter-antimatter asymmetry in the universe. Recently, a model has been proposed that can simultaneously explain these two pressing unknowns [1,2]. In this model, neutral $B$ mesons oscillate and then decay into a neutral dark-sector baryon (hereafter called $\psi_{\mathrm{DS}}$ ) plus SM

\footnotetext{
a e-mail: alexandre.brea.rodriguez@cern.ch

b e-mail: veronika.chobanova@cern.ch

c e-mail: xabier.cid.vidal@cern.ch (corresponding author)

de-mail: saul.lopez.solino@cern.ch

e e-mail: diego.martinez.santos@cern.ch

f e-mail: titus.mombacher@cern.ch

g e-mail: claire.prouve@cern.ch

h e-mail: emilioxose.rodriguez.fernandez@rai.usc.es

i e-mail: carlos.vazquez@cern.ch
}

hadrons. The matter anti-matter imbalance is then proportional [1] to

$$
\begin{aligned}
Y_{B} & \propto \sum_{s, d} A_{S L}^{s, d} \times \mathscr{B}\left(B_{s, d}^{0} \rightarrow \psi_{\mathrm{DS}} X\right) \\
= & -\sum_{s, d}\left|\frac{\Delta \Gamma_{s, d}}{\Delta m_{s, d} \cos \left(\phi_{s, d}-\arg \left(\Gamma_{12}^{s, d}\right)\right)}\right| \\
& \times \sin \left(\phi_{s, d}-\arg \left(\Gamma_{12}^{s, d}\right)\right) \times \mathscr{B}\left(B_{s, d}^{0} \rightarrow \psi_{\mathrm{DS}} X\right),
\end{aligned}
$$

where the semileptonic asymmetry is defined as

$$
A_{S L}^{s, d}=\frac{\Gamma\left(\bar{B}_{s, d}^{0} \rightarrow f\right)-\Gamma\left(B_{s, d}^{0} \rightarrow \bar{f}\right)}{\Gamma\left(\bar{B}_{s, d}^{0} \rightarrow f\right)+\Gamma\left(B_{s, d}^{0} \rightarrow \bar{f}\right)}
$$

with a final state $f(\bar{f})$ that is specific to $B_{s, d}^{0}\left(\bar{B}_{s, d}^{0}\right)$. It has been directly measured by several experiments, being the world averages $A_{S L}^{d}=(-21 \pm 17) \times 10^{-4}$ and $A_{S L}^{s}=(-6 \pm$ 28) $\times 10^{-4}$ [3]. Alternatively, it can be indirectly inferred through the $B_{s, d}^{0}$ mixing parameters $\Delta m_{s, d}, \Delta \Gamma_{s, d}, \phi_{s, d}$ and $\Gamma_{12}^{s, d}$. In the SM as well as in New Physics models with negligible contributions to $\Delta F=1$ penguins, these asymmetries are predicted to be $A_{S L}^{d}=(-4.73 \pm 0.42) \times 10^{-4}$ and $A_{S L}^{S}=(0.205 \pm 0.018) \times 10^{-4}[4]$, where similar values are found in Ref. [5]. Note that, since one needs a positive value for the semileptonic asymmetry in order to obtain a matterdominated universe, the possibility of having the baryogenesis mechanism lead by $B_{s}^{0}$ decays to dark-sector baryons is significantly favoured over those of $B^{0}$ mesons.

In order to fulfill the observed matter anti-matter imbalance, the inclusive branching fractions of $b$ hadrons decaying into the dark-sector baryon must be relatively large, $\gtrsim 10^{-4}$, with experimental upper limits ranging from $\leq 10^{-4}$ to $\leq 10^{-2}$ depending on the considered $\psi_{\text {DS }}$ mass $[2,6]$. Rel- 
atively high values could in principle also modify $\Gamma_{s} / \Gamma_{d}$ if they have different values for $B_{s}^{0}$ mesons compared to $B^{0}$ mesons. ${ }^{1}$

As for the exclusive branching fractions (as are the examples shown in this paper), these are expected to be at the $\gtrsim 10^{-6}$ level. Since the $\psi_{\text {DS }}$ baryon must be produced on shell in $B$-meson decays together with at least one proton (to preserve baryon number), its mass cannot be larger than $m_{B_{s}^{0}}-m_{p}$. In addition, to prevent the proton decay, the $\psi_{\mathrm{DS}}$ baryon must be heavier than the proton. Hence its mass range is limited to $940 \mathrm{MeV} / c^{2} \leq m_{\psi_{\mathrm{DS}}} \leq 4430 \mathrm{MeV} / c^{2}$. As an additional remark, we point the reader to Refs. [7,8], which approach the same problem from slightly different angles.

Due to its unique design and the abundant production of $b$ hadrons in high-energetic $p p$ collisions, the $\mathrm{LHCb}$ experiment is ideally suited for searching for the proposed type of baryonic dark matter in $b$-hadron decays $[9,10]$. Equipped with a highly granular vertex detector very close to the $p p$-interaction point [11], a precise reconstruction of the $b$-hadron decay vertex can be achieved, which is crucial to obtain a good sensitivity in the search for the dark baryon. Due to baryon-number conservation, searches using $B$ mesons need at least one proton and at least another charged particle to reconstruct the decay vertex. This implies $B$ mesons alone cannot cover the entire phase space of the model. However, $\mathrm{LHCb}$ can profit from its large production of $b$ baryons, which do not require the presence of a proton in the final state to preserve baryon number, and which are also heavier than $B_{s}^{0}$ mesons.

In this paper, we use as benchmark modes the decays $B^{0} \rightarrow \psi_{\mathrm{DS}} \Lambda(1520)\left(\rightarrow p K^{-}\right), B^{+} \rightarrow \psi_{\mathrm{DS}} \Lambda_{c}(2595)^{+}(\rightarrow$ $\left.\pi^{+} \pi^{-} \Lambda_{c}^{+}\left(\rightarrow p K^{-} \pi^{+}\right)\right), \Lambda_{b}^{0} \rightarrow \psi_{\mathrm{DS}} K^{+} \pi^{-}$, and $\Lambda_{b}^{0} \rightarrow$ $\psi_{\mathrm{DS}} \pi^{+} \pi^{-2}$ for the search of the dark baryon $\psi_{\mathrm{DS}}$ with the $\mathrm{LHCb}$ experiment. These channels are sensitive to different $\mathscr{O}_{q_{1} q_{2}}$ operators out of those introduced in Refs. [1,2]. More precisely, $B^{0} \rightarrow \psi_{\mathrm{DS}} \Lambda(1520)$ decays would be sensitive to $\mathscr{O}_{u s}, B^{+} \rightarrow \psi_{\mathrm{DS}} \Lambda_{c}(2595)^{+}$to $\mathscr{O}_{c d}, \Lambda_{b}^{0} \rightarrow \psi_{\mathrm{DS}} K^{+} \pi^{-}$ to $\mathscr{O}_{u s}$, and $\Lambda_{b}^{0} \rightarrow \psi_{\mathrm{DS}} \pi^{+} \pi^{-}$to $\mathscr{O}_{u d}$. Since none of these operators are favored a priori by the mechanism, it becomes essential to probe as many of them as possible.

We explore the possibility of further background suppression by tagging some of these decays via the decay chains $\Sigma_{b}^{(*) \pm} \rightarrow \Lambda_{b}^{0} \pi^{ \pm}$. The projections are based on the detector geometry proposed for the data taking periods Run 3 and Run 4 of the LHCb experiment [12] and its Upgrade II [13]. The expected sensitivities are computed at corresponding benchmark luminosities of 15 (Run 3), 50 (Run 4), and $300 \mathrm{fb}^{-1}$ (Upgrade II) [13] of $p p$ collisions at a center-of-mass energy of $14 \mathrm{TeV}$.

\footnotetext{
${ }^{1}$ G. Alonso, private communication.

${ }^{2}$ Charge-conjugate modes are included unless explicitly stated.
}

The document is organised as follows: in Sect. 2 we describe the tools used to simulate events and specific decays within the LHCb detector; Sect. 3 discusses potential event selections; and in Sect. 4 we describe the estimated sensitivities for the different modes; finally, potential systematic effects are discussed in Sect. 5; we conclude in Sect. 6.

\section{Event generation and simulation}

Proton-proton collisions are simulated with PYTHIA 8.226 [14] at a center-of-mass energy of $14 \mathrm{TeV}$. Note that this does not include $B_{c}^{+}$mesons and therefore backgrounds from $B_{c}^{+}$ mesons are not considered in this study.

The four-momenta and origin point of the particles of interest that have been produced by PYTHIA are stored for further processing. Subsequently, signal $b$ hadrons are decayed according to phase space. No bremsstrahlung is included in the generation of the decays. Background events are obtained from $b \bar{b}$ events in PYTHIA filtered with selection cuts before proceeding with detector simulation, in order to reduce the amount of events to process.

To obtain the background and signal yields, we use the $b \bar{b}$ cross sections as measured by the LHCb experiment at $\sqrt{s}=13 \mathrm{TeV}$ and $\sqrt{s}=7 \mathrm{TeV}$ [15] extrapolated linearly with the center-of-mass energy to $\sqrt{s}=14 \mathrm{TeV}$. Hadronisation fractions for $b$ hadrons are obtained from Refs. [15, 16], assuming $f_{u}=f_{d}$. The production of heavier $b$ baryons and $b$ hadrons with multiple heavy quarks is neglected. We also neglect the effect of pile-up, given the dominant background is composed of single $b \bar{b}$ events and also that we expect the effect of wrong primary vertex association ${ }^{3}$ to barely affect the sensitivity, given the future vertexing detector of $\mathrm{LHCb}$ will be 4D [17] (including timing), which is known to be very useful to mitigate this effect.

The detector is simulated using the code in Ref. [9]. The simulated detector elements are the RF foil, the VeloPix (VP) stations, the Upstream Tracker, the Magnet, and the SciFi Tracker $[11,18]$, and are implemented in python2. No calorimetry or particle identification (PID) is simulated at this stage. The particles generated by the procedure described above are passed through the detector elements and yield hits where appropriate. The set of hits is then processed by a track fit algorithm which calculates the track slopes at origin, the momentum, and a point in the early stage of the particle trajectory. Note that through this procedure all detector acceptance effects are accounted for. The simulation neglects occupancy effects and hit inefficiency. To account for this, when obtaining absolute efficiencies, the tracking efficiency is arti-

\footnotetext{
${ }^{3}$ I.e. to associate the signal candidate to a wrong primary vertex, which potentially affects the determination of variables such the impact parameter.
} 


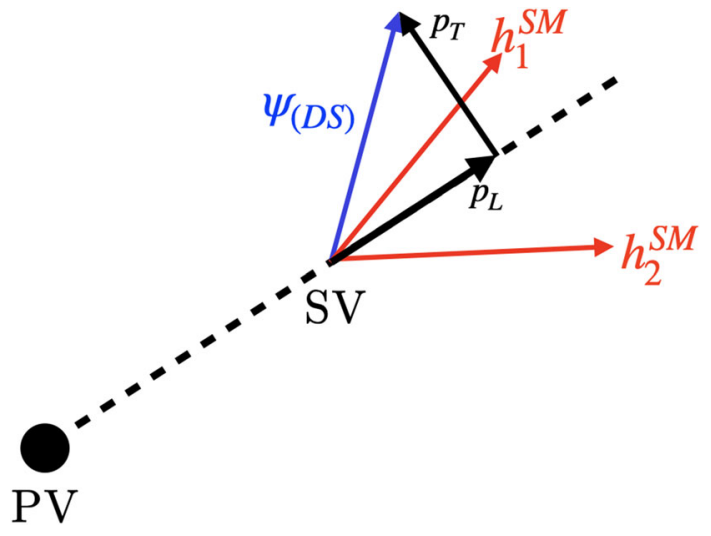

Fig. 1 Determination of $p_{\mathrm{T}}^{\mathrm{miss}}$ in $X_{b} \rightarrow \psi_{\mathrm{DS}} h_{1}^{S M} h_{2}^{S M}$ decays. For the $B^{+} \rightarrow \psi_{\mathrm{DS}} \Lambda_{c}(2595)^{+}$channel, where more objects are present in the final state, the computation would be analogous. In the figure, PV (SV) represent the primary (secondary) vertices, i.e., the $b$-hadron production (decay) points

ficially scaled by $98 \%$. The detector simulation reproduces at first order the impact parameter and momentum resolutions of the existing full simulation of the LHCb Upgrade, as well as their kinematic dependencies $[11,18]$.

The final computation of the expected exclusions for the decay channels under evaluation is performed by means of the "missing" transverse momentum $\left(p_{\mathrm{T}}^{\text {miss }}\right)$. This is defined as the sum of all the momenta of the reconstructed daughters in the direction transverse to the $b$-hadron direction of flight, as illustrated in Fig. 1. This quantity can only be determined if both the $b$-hadron origin and decay position are known. Both of these properties can be computed experimentally, since they correspond to the $p p$-interaction point, or the origin of all the final-state charged tracks of the $b$ hadron, respectively. The requirement to know the $b$-hadron decay position limits the amount of channels one can study, since this involves knowing the trajectory of the final state SM daughters, which is not possible if these are stable or very long-lived neutral particles. The $p_{\mathrm{T}}^{\text {miss }}$ variable is used in our study to discriminate signal from background. For the $B \rightarrow \psi_{\mathrm{DS}} \Lambda$ analyses, the actual expected limit is determined based on this quantity. Furthermore, for the $\Lambda_{b}^{0} \rightarrow \psi_{\mathrm{DS}} h_{1}^{+} h_{2}^{-}$analyses, also the $h_{1}^{+} h_{2}^{-}$invariant mass, $m\left(h_{1}^{+} h_{2}^{-}\right)$, is very useful since it shows sharp kinematic end points at the $\psi_{\text {DS }}$ mass [9], so the limit determination is based on a region selected in the two-dimensional $\left(m\left(h_{1}^{+} h_{2}^{-}\right)-p_{\mathrm{T}}^{\text {miss }}\right)$ plane.

\section{Event selection}

All tracks involved in the analysis are required to satisfy $p_{\mathrm{T}}>800 \mathrm{MeV} / c$ and $0.1<\mathrm{IP}<3 \mathrm{~mm}$, where $p_{\mathrm{T}}$ is the transverse momentum in the laboratory frame and IP the impact parameter with respect to the $p p$ interaction vertex. ${ }^{4}$ The only exception to this are the pions appearing in the $B^{+} \rightarrow \psi_{\mathrm{DS}} \Lambda_{c}(2595)^{+}$decay for which the $p_{\mathrm{T}}$ cut is relaxed to $250 \mathrm{MeV} / c$. We assume $100 \%$ trigger efficiency.

The rest of the cuts are channel dependent and are explained in the next subsections.

\subsection{Isolation}

The main background for the targeted decay channels in this analysis is composed of hadrons coming from a $b \bar{b}$ pair. A key feature of this situation is that the background tracks are typically accompanied by other objects, while for the signal the final state is "isolated" and no other nearby particles are expected. Based on this feature, we build a quantity that provides discrimination between signal and background. For this, we take all charged particles arising from $b \bar{b}$ pairs (having excluded those forming the signal candidates), and we select only those reconstructed using our fast simulation procedure, with $p_{\mathrm{T}}>250 \mathrm{MeV} / c$. With this, and following Refs. [19,20], we determine the smallest distance of closest approach (DOCA) to our candidates and use that to measure the isolation. The distribution of this quantity, DOCA ISO will peak at smaller quantities for background than for signal. The cut we apply, DOCA ISO $>0.05$, retains $\sim 80 \%$ of signal candidates and suppresses $\sim 75 \%$ of the background.

\subsection{Selection for $B \rightarrow \psi_{\mathrm{DS}} \Lambda$ decays}

The selection of each $B \rightarrow \psi_{\mathrm{DS}} \Lambda$ decay modes is based on the full reconstruction of the $\Lambda$ resonances, applying invariant mass and DOCA cuts to the relevant daughter particles. Furthermore, IP and $p_{T}$ cuts are applied to the $\Lambda$ candidates. The full list of cuts for these two channels can be found in Table 1, and the main considerations for each of the selections in the next paragraphs.

For the $B^{0} \rightarrow \psi_{\mathrm{DS}} \Lambda(1520)$ decay mode, the $\Lambda(1520)$ resonance is reconstructed through its decay to a $p K^{-}$pair. Each member of the pair is required to be close to each other (applying a DOCA cut), and an invariant mass cut to the pair is also applied, so the mother candidate has an invariant mass consistent with that of the $\Lambda(1520)$. Furthermore, the $\Lambda$ (1520) candidate must not be consistent with originating at the $p p$ interaction vertex (IP cut) and satisfy a minimum $p_{T}$ cut.

For the $B^{+} \rightarrow \psi_{\mathrm{DS}} \Lambda_{c}(2595)^{+}$channel, two subsequent decays are required to reconstruct the signal candidates, $\Lambda_{c}(2595)^{+} \rightarrow \Lambda_{c}^{+} \pi^{+} \pi^{-}$and $\Lambda_{c}^{+} \rightarrow p K^{-} \pi^{+}$. After applying the track-level cuts described above, the $\Lambda_{c}^{+}$candidates

\footnotetext{
4 The lower IP cut discriminates against particles produced at the $p p$ interaction vertex, while the IP upper cut helps to remove potential ghost tracks.
} 
are assumed to be very clean and no further cuts are applied to them. No fake combinations of $\Lambda_{c}^{+}$are considered either, since this is a very clean resonance at $\mathrm{LHCb}$, being narrow in invariant mass, displaced ${ }^{5}$ and with a final state including a proton, a kaon and a pion, which are separable from each other through PID cuts. Real reconstructed $\Lambda_{c}^{+}$baryons are then combined with pions to form $\Lambda_{c}(2595)^{+}$candidates. Then, as in the $\Lambda$ (1520) case, invariant mass, DOCA, IP and $p_{T}$ cuts are applied to these.

One relevant exclusive background we have considered for both channels is that originating from $X_{b} \rightarrow$ $Y_{\mathrm{SM}} \Lambda_{c}(2595)^{+}$and $X_{b} \rightarrow Y_{\mathrm{SM}} \Lambda$ (1520) decays, i.e., cases in which the baryons accompanying $\psi_{\mathrm{DS}}$ are actually produced in $b$-hadron $\left(X_{b}\right)$ SM decays together with other SM particles $\left(Y_{\mathrm{SM}}\right)$. Although closer to signal than the combinatorial background, these type of candidates are still separable by means of the isolation and $p_{\mathrm{T}}^{\text {miss }}$. After all selection cuts, they are seen to contribute $<0.5 \%$ compared to the combinatorial background for the $B^{0} \rightarrow \psi_{\mathrm{DS}} \Lambda$ (1520) channel, so are not considered further in this case. On the contrary, for the $B^{+} \rightarrow \psi_{\mathrm{DS}} \Lambda_{c}(2595)^{+}$channel, they are relevant and become the dominant source of background, so they are accounted for in the sensitivity determination. The main contribution to this type of background in this channel is composed of semileptonic $\Lambda_{b}$ decays, i.e $\Lambda_{b} \rightarrow \Lambda_{c}(2595)^{ \pm} l^{\mp} \nu_{l}$, with $l^{\mp}$ and $\nu_{l}$ being a charged lepton and corresponding neutrino, respectively.

\subsection{Selection and multivariate classifier for} $\Lambda_{b}^{0} \rightarrow \psi_{\mathrm{DS}} h_{1}^{+} h_{2}^{-}$decays

The $\Lambda_{b}^{0} \rightarrow \psi_{\mathrm{DS}} h_{1}^{+} h_{2}^{-}$decays suffer similarly from a large amount of combinatorial background from $b \bar{b} \rightarrow h_{1}^{+} h_{2}^{-} X$ processes. In order to improve the discrimination power of the analysis, multivariate classifiers are built using the $p_{\mathrm{T}}^{\text {miss }}$ and the $h_{1}^{+} h_{2}^{-}$reconstructed mass as input variables. Before the training, the following cuts are applied: the two meson tracks must satisfy the cuts introduced at the end of Sect. 2, the DOCA between the two mesons is required to be less than $0.1 \mathrm{~mm}$, the distance of flight in the $\mathrm{z}$ direction $(\Delta z)$ larger than $5 \mathrm{~mm}$, the transverse momentum of the track larger than $1 \mathrm{GeV} / c$, and the ratio between the impact parameter of the meson pair and the distance of flight in the $\mathrm{z}$ direction $\left(\operatorname{IP}\left(h_{1}^{+} h_{2}^{-}\right) / \Delta z\right)$ is required to be smaller than 0.1 . The classifier relies on the mathematical method used in Ref. [22] (see also Ref. [23]). A cut is performed in the multivariate classifier response such that it minimizes the expected limit at a given integrated luminosity and $\psi_{\text {DS }}$ mass.

\footnotetext{
5 In this case there is a "double" displacement given these $\Lambda_{c}^{+}$are produced in $B$-meson decays.
}

\subsection{Efficiencies}

The efficiencies of the selection cuts proposed in this paper are shown in Table 2 for different benchmark masses of the dark baryon, $m_{\psi_{\mathrm{DS}}}$. We also simulate the effect of applying PID cuts that would make the background contribution from misidentified particles negligible. For kaons, we take the expected kaon identification efficiency for a Delta Log Likelihood (DLL) DLL $_{K \pi}>5$ cut as a function of momentum [24], and apply it to our samples, which provides a penalty efficiency factor. For protons we follow a similar procedure, applying DLL $>5$ cuts to discriminate protons against kaons and pions. In this case, since no projections exist for the $\mathrm{LHCb}$ upgrades, we take the measured numbers from the data-taking periods Run 1 and Run 2 of the $\mathrm{LHCb}$ experiment [25].

\subsection{Tagging}

A useful way to deal with background in $\Lambda_{b}^{0}$ decays while getting additional kinematic information, is to select those $\Lambda_{b}^{0}$ particles coming from $\Sigma_{b}^{(*) \pm}$ as suggested in Ref. [26]. About one third of $\Lambda_{b}^{0}$ baryons are coming from $\Sigma_{b}^{(*) \pm}$ according to PYTHIA [14]. More importantly, from Ref. [27] one can infer that $\mathrm{LHCb}$ yields approximately one tagged $\Lambda_{b}^{0}$ per 10 untagged $\Lambda_{b}^{0}$, including the effect of reconstructing and selecting the associated slow pion from the decay $\Sigma_{b}^{(*) \pm} \rightarrow$ $\Lambda_{b}^{0} \pi^{ \pm}$. Most background events will have plenty of slow prompt pions coming from the same collision point as the $\Lambda_{b}^{0}$ candidate. However, background events are not expected to peak at the $\Sigma_{b}^{(*)}$ mass, while signal events do (see Fig. 2). This could allow extra selection cuts for further background reduction, as well as a good signal confirmation due to the distinctive two-peak pattern. In these sensitivity studies the usage of tagging is not included, though.

As for the $B \rightarrow \psi_{\mathrm{DS}} \Lambda$ modes, tagging would also be possible in principle for the $B^{+} \rightarrow \psi_{\mathrm{DS}} \Lambda_{c}(2595)^{+}$channel through $B^{+}$mesons produced in $B_{s 2}^{* 0} \rightarrow B^{+} K^{-}$decays. Around $20 \%$ of all $B^{+}$mesons are produced in a $B_{s 2}^{* 0}$ decay, according to PYTHIA. Note that this type of tagging has already been used at $\mathrm{LHCb}$ to search for the lepton flavor violating $B^{+} \rightarrow K^{+} \mu^{-} \tau^{+}$decay [28], where the $\tau$ lepton was effectively treated as a missing particle. This search achieved upper limits on the branching fraction of this decay at the level of $10^{-5}$, using an $\mathrm{LHCb}$ data set accounting for an integrated luminosity of $9 \mathrm{fb}^{-1}$.

\section{Estimated statistical sensitivities}

As discussed in Sect. 2, we obtain the sensitivities from the distributions of $p_{\mathrm{T}}^{\text {miss }}$, or $p_{\mathrm{T}}^{\text {miss }}$ versus $m\left(h_{1}^{+} h_{2}^{-}\right)$for signal 
Table 1 Requirements for each $B \rightarrow \psi_{\mathrm{DS}} \Lambda$ decay mode. These values have been chosen in order to maximize the discrimination between signal and background. Here, "max(DOCA)" refers to the maximum value of all the DOCA computed for the two-track combinations among the $\Lambda(1520)$ and $\Lambda_{c}(2595)^{+}$daughters, and $\left|m_{\Lambda}-m_{\mathrm{PDG}}\right|$ describes the mass window in the relevant reconstructed invariant $\Lambda$ mass around its known mass [21]. The IP and $p_{\mathrm{T}}$ cuts are applied to the $\Lambda(1520)$ and $\Lambda_{c}(2595)^{+}$candidates. All the track cuts, introduced in Sect. 2, have also been imposed for every decay mode

\begin{tabular}{lll}
\hline Variable & $B^{+} \rightarrow \psi_{\mathrm{DS}} \Lambda_{c}(2595)^{+}$ & $B^{0} \rightarrow \psi_{\mathrm{DS}} \Lambda(1520)$ \\
\hline DOCA ${ }_{\text {ISO }}$ & $0.05 \mathrm{~mm}$ & $0.05 \mathrm{~mm}$ \\
$\max (\mathrm{DOCA})$ & $0.15 \mathrm{~mm}$ & $0.075 \mathrm{~mm}$ \\
$\left|m_{\Lambda}-m_{\mathrm{PDG}}\right|$ & $20 \mathrm{MeV} / c^{2}$ & $7.5 \mathrm{MeV} / \mathrm{c}^{2}$ \\
$\mathrm{IP}$ & $0.07 \mathrm{~mm}$ & $0.1 \mathrm{~mm}$ \\
$p_{\mathrm{T}}$ & $4000 \mathrm{MeV} / c$ & $1000 \mathrm{MeV} / c$ \\
\hline
\end{tabular}

Table 2 Reconstruction and selection efficiencies for the signal decays described in the text, as obtained from fast simulation, where we added a posteriori a $98 \%$ efficiency per track to account for multiplicity effects. Geometry of the detector is considered within $\varepsilon^{R E C}$. The efficiencies are shown in $\%$. The $\varepsilon^{R E C \& P T}$ efficiency implies that all tracks have been required to have a transverse momentum greater than $800 \mathrm{MeV} / c$, except for $B^{+} \rightarrow \psi_{\mathrm{DS}} \Lambda_{c}(2595)^{+}$decays, where this requirement is relaxed to $250 \mathrm{MeV} / c$ for pions. The $\varepsilon^{S E L / R E C}$ efficiency includes multivariate and isolation cuts when applicable. $\varepsilon^{P I D / S E L}$ refers to the efficiency assumed for the PID cuts, as explained in the text. Note this does not apply to modes with no protons or kaons in the final state. Finally, $\varepsilon^{\text {TOTAL }}$ is the product of $\varepsilon^{R E C}, \varepsilon^{S E L / R E C}$ and $\varepsilon^{P I D / S E L}$. The number next to $\psi_{\mathrm{DS}}$ refers to $m_{\psi_{\mathrm{DS}}}$, given in $\mathrm{MeV} / c^{2}$

\begin{tabular}{|c|c|c|c|c|c|}
\hline Decay mode & $\varepsilon^{R E C}$ & $\varepsilon^{R E C \& P T}$ & $\varepsilon^{S E L / R E C}$ & $\varepsilon^{P I D / S E L}$ & $\varepsilon^{T O T A L}$ \\
\hline$\Lambda_{b}^{0} \rightarrow \psi_{\mathrm{DS}}(940) K^{+} \pi^{-}$ & 7.6 & 5.1 & 13.2 & 74.2 & 0.74 \\
\hline$\Lambda_{b}^{0} \rightarrow \psi_{\mathrm{DS}}(940) \pi^{+} \pi^{-}$ & 7.3 & 4.8 & 14.3 & - & 1.04 \\
\hline$\Lambda_{b}^{0} \rightarrow \psi_{\mathrm{DS}}(1500) K^{+} \pi^{-}$ & 7.7 & 4.8 & 10.8 & 76.0 & 0.63 \\
\hline$\Lambda_{b}^{0} \rightarrow \psi_{\mathrm{DS}}(1500) \pi^{+} \pi^{-}$ & 7.3 & 4.5 & 12.5 & - & 0.91 \\
\hline$\Lambda_{b}^{0} \rightarrow \psi_{\mathrm{DS}}(2000) K^{+} \pi^{-}$ & 7.7 & 4.5 & 6.67 & 79.2 & 0.41 \\
\hline$\Lambda_{b}^{0} \rightarrow \psi_{\mathrm{DS}}(2000) \pi^{+} \pi^{-}$ & 7.4 & 4.1 & 10.9 & - & 0.81 \\
\hline$\Lambda_{b}^{0} \rightarrow \psi_{\mathrm{DS}}(2400) K^{+} \pi^{-}$ & 7.8 & 4.1 & 9.11 & 80.8 & 0.57 \\
\hline$\Lambda_{b}^{0} \rightarrow \psi_{\mathrm{DS}}(2400) \pi^{+} \pi^{-}$ & 7.4 & 3.7 & 8.89 & - & 0.66 \\
\hline$\Lambda_{b}^{0} \rightarrow \psi_{\mathrm{DS}}(4340) K^{+} \pi^{-}$ & 8.1 & 1.2 & 3.12 & 88.0 & 0.22 \\
\hline$\Lambda_{b}^{0} \rightarrow \psi_{\mathrm{DS}}(4340) \pi^{+} \pi^{-}$ & 7.4 & 0.97 & 2.88 & - & 0.21 \\
\hline$\Lambda_{b}^{0} \rightarrow \psi_{\mathrm{DS}}(4470) K^{+} \pi^{-}$ & 8.2 & 0.91 & 2.18 & 87.7 & 0.16 \\
\hline$\Lambda_{b}^{0} \rightarrow \psi_{\mathrm{DS}}(4470) \pi^{+} \pi^{-}$ & 7.4 & 0.79 & 2.23 & - & 0.17 \\
\hline$B^{+} \rightarrow \psi_{\mathrm{DS}}(940) \Lambda_{c}(2595)^{+}$ & 5.7 & 1.3 & 20.9 & 56.7 & 0.68 \\
\hline$B^{+} \rightarrow \psi_{\mathrm{DS}}(1500) \Lambda_{c}(2595)^{+}$ & 5.6 & 1.1 & 19.3 & 56.6 & 0.61 \\
\hline$B^{+} \rightarrow \psi_{\mathrm{DS}}(2000) \Lambda_{c}(2595)^{+}$ & 5.3 & 0.88 & 18.5 & 57.2 & 0.56 \\
\hline$B^{+} \rightarrow \psi_{\mathrm{DS}}(2400) \Lambda_{c}(2595)^{+}$ & 4.9 & 0.66 & 16.4 & 57.1 & 0.46 \\
\hline$B^{0} \rightarrow \psi_{\mathrm{DS}}(940) \Lambda(1520)$ & 13.3 & 12.7 & 38.1 & 56.9 & 2.88 \\
\hline$B^{0} \rightarrow \psi_{\mathrm{DS}}(1500) \Lambda(1520)$ & 13.7 & 12.9 & 36.6 & 56.4 & 2.83 \\
\hline$B^{0} \rightarrow \psi_{\mathrm{DS}}(2000) \Lambda(1520)$ & 13.5 & 12.6 & 35.5 & 56.8 & 2.72 \\
\hline$B^{0} \rightarrow \psi_{\mathrm{DS}}(2400) \Lambda(1520)$ & 13.3 & 6.8 & 34.5 & 56.6 & 2.6 \\
\hline$B^{0} \rightarrow \psi_{\mathrm{DS}}(3500) \Lambda(1520)$ & 12.1 & 3.5 & 23.6 & 56.5 & 1.61 \\
\hline
\end{tabular}

and background candidates surviving the selections introduced in Sect. 3. In order to maximize our sensitivity, the number of observed events in background of the $B$-meson decay modes are obtained in bins of $p_{\mathrm{T}}^{\text {miss }}$, while for the $\Lambda_{b}^{0}$ decay modes a single two-dimensional region as a function of $p_{\mathrm{T}}^{\text {miss }}$ and $m\left(h_{1}^{+} h_{2}^{-}\right)$is considered. Figures 3 and 4 show the background and signal distributions (for different values of the dark baryon mass) for the $B \rightarrow \psi_{\mathrm{DS}} \Lambda$ and $\Lambda_{b}^{0} \rightarrow \psi_{\mathrm{DS}} h_{1} h_{2}$ decay modes, respectively.

Exclusion limits on the decay branching fractions of the four $B$ and $\Lambda_{b}^{0}$ decay modes are then computed using these distributions, with the Modified Frequentist confidence level $\left(\mathrm{CL}_{s}\right)$ method, described in Refs. [29,30]. These values are obtained per decay mode and benchmark $\psi_{\text {DS }}$ mass hypotheses as listed in Table 2. 

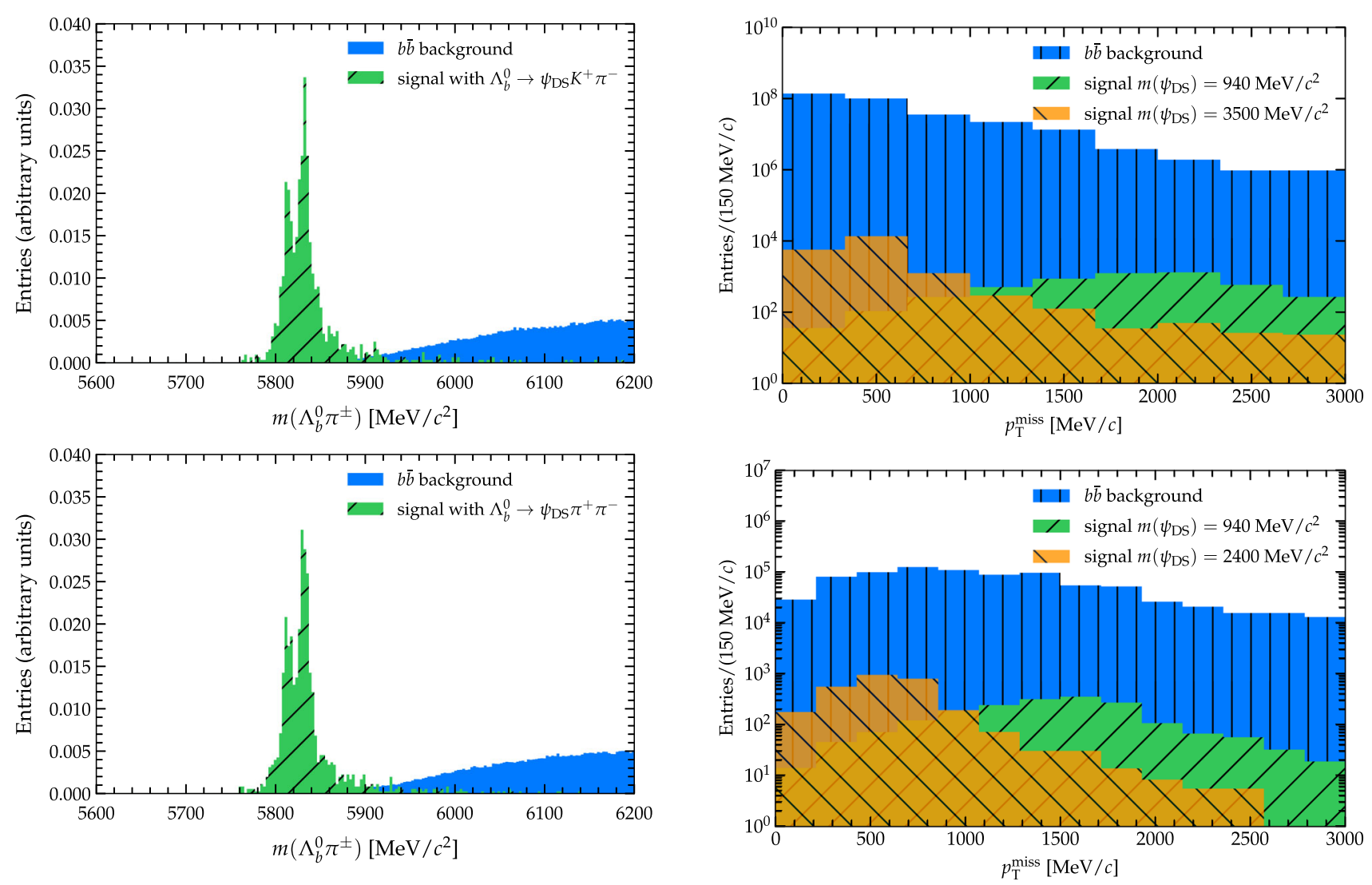

Fig. 2 Normalised distributions of the reconstructed invariant mass of $\Sigma_{b}^{(*) \pm} \rightarrow \Lambda_{b}^{0}\left(\rightarrow \psi_{\mathrm{DS}} h_{1}^{+} h_{2}^{-}\right) \pi^{ \pm}$candidates in the simulated samples. The mass of the $\Lambda_{b}$ baryon is a fixed parameter in this case. Hatched green: signal, blue: $b \bar{b}$ background. Top: $\Lambda_{b}^{0} \rightarrow \psi_{\mathrm{DS}} K^{+} \pi^{-}$candidates. Bottom: $\Lambda_{b}^{0} \rightarrow \psi_{\mathrm{DS}} \pi^{+} \pi^{-}$candidates. The tagging of $\Lambda_{b}^{0}$ allows a clear distinction between signal and background samples

Finally, we consider the limits at 95\% CL per $\psi_{\text {DS }}$ mass hypothesis and we interpolate these values to cover intermediate mass points, as presented in Fig. 5. For $B$-meson decay modes an exponential extrapolation is used for masses above $3.5(2.5) \mathrm{GeV} / \mathrm{c}^{2}$, to account for the inability to reconstruct the $\Lambda$ mesons due to the limited phase space.

For all the decay modes, branching fractions down to (1$5) \times 10^{-6}$ for a dark baryon mass between 1 and $2.5 \mathrm{GeV} / \mathrm{c}^{2}$, could be excluded. The best limits are achieved in the low $m_{\psi_{\text {DS }}}$ region and are given by the $B^{0}$ mode we used, because of the complex final state. High masses are only accessible with $\Lambda_{b}^{0}$ decays. It should be noted that at high masses the differences between the inclusive and exclusive branching fraction are smaller, and hence higher branching fractions are expected.

\section{Systematic uncertainties}

It should be noted that in these analyses, the background yields are typically larger than in other searches, such as

Fig. 3 Distributions of $p_{\mathrm{T}}^{\text {miss }}$ for $B \rightarrow \psi_{\mathrm{DS}} \Lambda$ and associated backgrounds. Top: $B^{0} \rightarrow \psi_{\mathrm{DS}} \Lambda(1520)$. Bottom: $B^{+} \rightarrow \psi_{\mathrm{DS}} \Lambda_{c}(2595)^{+}$. Blue: background from inclusive $b \bar{b}$ events. Orange: signal for a high mass (close to phase space limit) dark baryon. Green: signal for a dark baryon at $940 \mathrm{MeV} / c^{2}$. The signal and background yields correspond to those expected at $\mathrm{LHCb}$ at $15 \mathrm{fb}^{-1}$, with the signal branching fractions assumed to be those that could be excluded at 95\% CL (see Sect. 4). No systematic uncertainties are assumed in these plots

rare decays. Hence, systematic uncertainties related to background yield or modelling can potentially limit the search. For instance, with a background yield systematic uncertainty of $1 \%$ all the searches shown in this study would be already systematically limited with $1 \mathrm{fb}^{-1}$, and with a systematic uncertainty of 1 per mile, they would be systematically limited after $\sim 15 \mathrm{fb}^{-1}$. Hence, further background suppression, such as usage of the $\Sigma_{b}^{(*)}$ (or $B_{s 2}^{* 0}$ ) tagging, more complex decay chains or the use of control regions might be needed to perform the proposed searches at significantly higher integrated luminosity. The presence of systematic uncertainties might also risk reaching the whole region of theoretical interest, with branching fractions $\gtrsim 10^{-5}$ for the channels of interest and any value of $m_{\psi_{\mathrm{DS}}}$. Background modeling will of course be particularly crucial when $m_{\psi_{\mathrm{DS}}}$ approaches the mass of an existing SM resonance, since isolation cuts only reduce the backgrounds from $b \bar{b}$ pairs, but do not grant that the remaining signal candidates are not coming from exclu- 

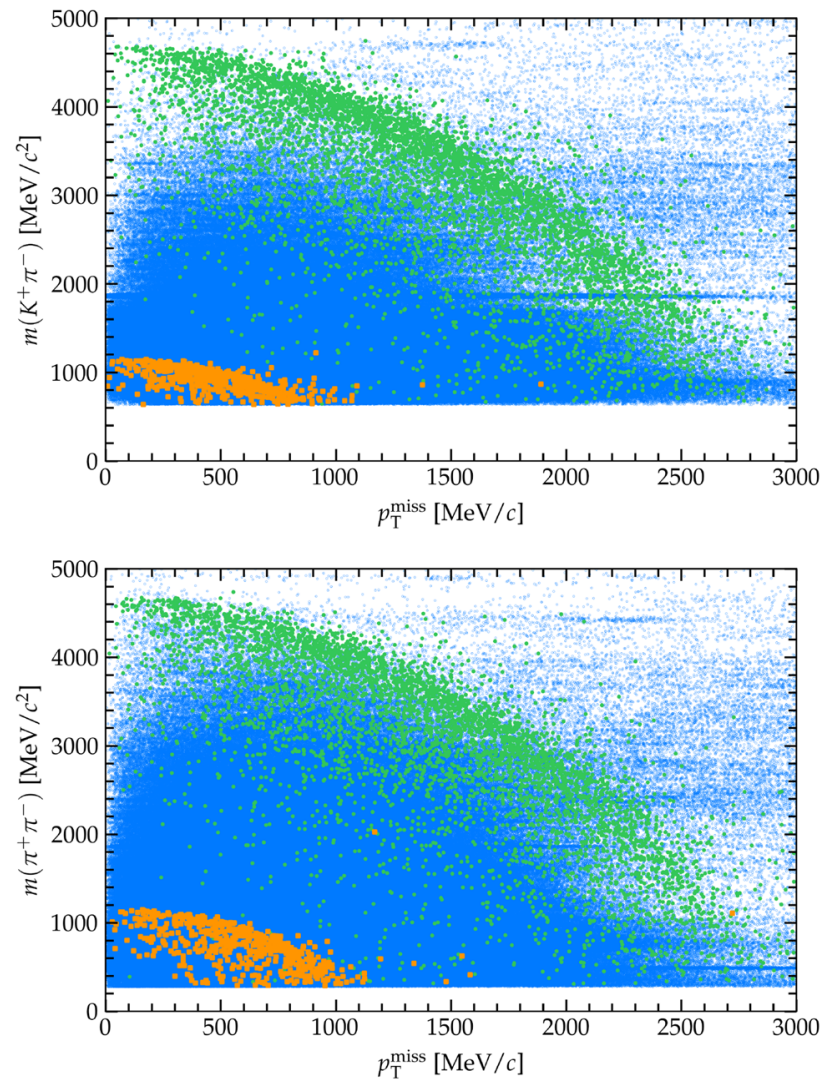

Fig. 4 Reconstructed invariant mass versus $p_{\mathrm{T}}^{\text {miss }}$ for $\Lambda_{b}^{0} \rightarrow \psi_{\mathrm{DS}} h_{1}^{+} h_{2}^{-}$ candidates. Top: $\Lambda_{b}^{0} \rightarrow \psi_{\mathrm{DS}} K^{+} \pi^{-}$. Bottom: $\Lambda_{b}^{0} \rightarrow \psi_{\mathrm{DS}} \pi^{+} \pi^{-}$. Blue dots: background from inclusive $b \bar{b}$ events, green circles: signal for a dark baryon at $940 \mathrm{MeV} / c^{2}$, orange squares: signal for a dark baryon at $4470 \mathrm{MeV} / c^{2}$. Background from light mass resonances, such as $K^{*}(892) \rightarrow K^{+} \pi^{-}, D^{0} \rightarrow K^{+} \pi^{-}$, and $K_{S}^{0} \rightarrow \pi^{+} \pi^{-}$is clearly visible in the plots. No systematic uncertainties are assumed in these plots

sive backgrounds. More details about the effect of systematic uncertainties are explained in Appendix A.

\section{Conclusions}

The B-mesogenesis mechanism predicts branching fractions of $b$-hadron decays to dark baryons at relatively large values $\left(\sim 10^{-2}-10^{-6}\right)$. The closer the CP-violation sources are to the SM prediction, the higher those branching fractions need to be in order to fulfill the baryon asymmetry of the universe. In our study, we show that LHCb Upgrade will have the statistical sensitivity to search for branching fractions at the $10^{-5}$ level or better for the entire mass range of the dark baryon $\psi_{\text {DS }}$. Multi-body final states, such as $B^{+} \rightarrow$ $\psi_{\text {DS }}(940) \Lambda_{c}(2595)^{+}$, show the best performance at lower values of $m_{\psi_{\mathrm{DS}}}$ while two-track final states are needed to reach higher masses, with $\Lambda_{b}^{0}$ decays allowing to test the full allowed mass range. Systematic uncertainties could play
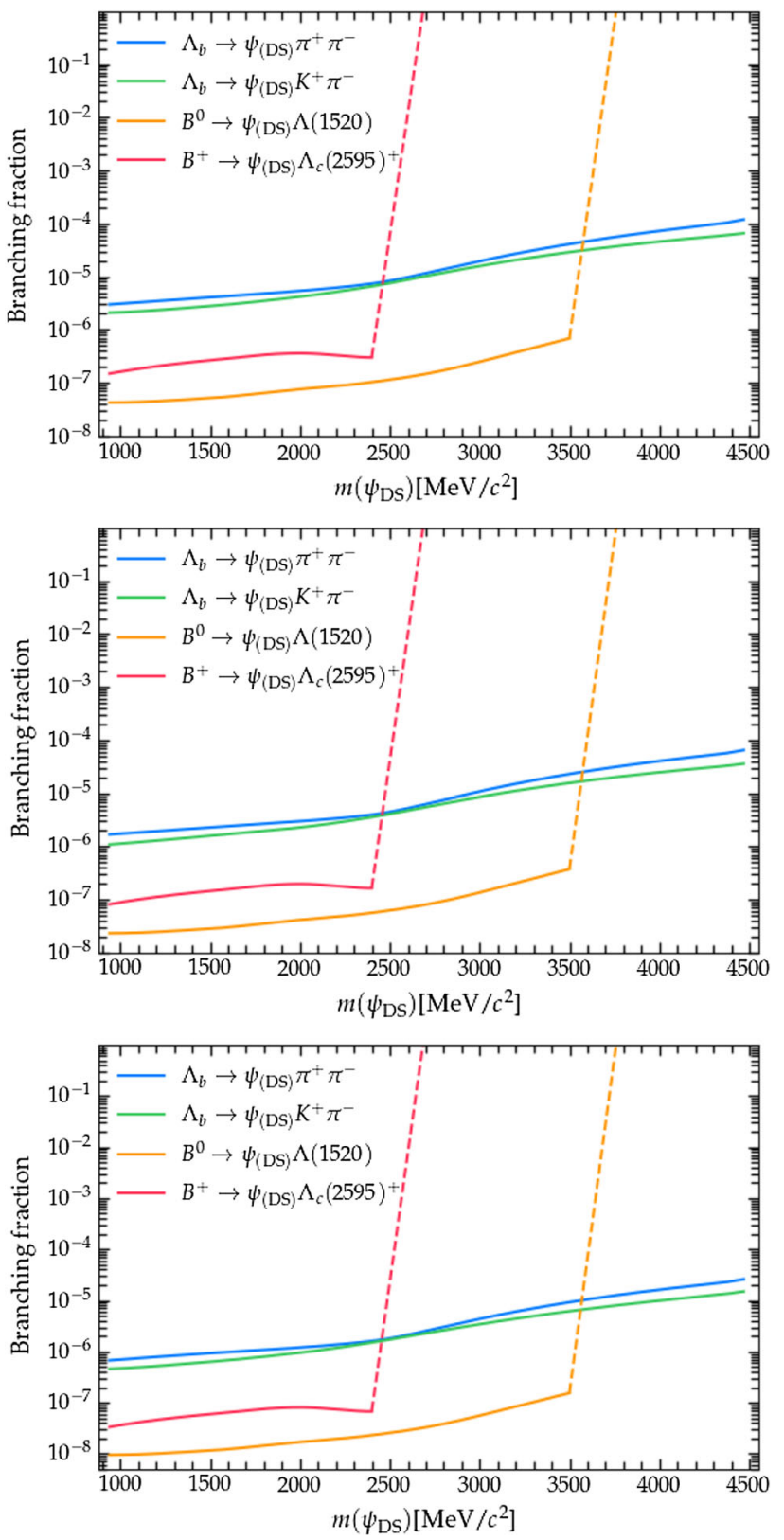

Fig. 5 Projected statistical sensitivity in terms of branching fractions excluded at $95 \% \mathrm{CL}$ at, from top to bottom, $15 \mathrm{fb}^{-1}, 50 \mathrm{fb}^{-1}$ and $300 \mathrm{fb}^{-1}$. All three curves were obtained by interpolation, having used an exponential extrapolation (dashed line) in the abrupt change of slope for the $B$ decays in order to account for the detector's inability to reconstruct neither the $\Lambda(1520)$ nor the $\Lambda_{c}(2595)^{+}$baryons at the limit of the phase space, i.e., whenever $m_{\psi_{\mathrm{DS}}}$ reaches the mass difference of the SM mother meson and daughter baryon

an important role in the sensitivity, and hence very precise background modelling and suppression could be needed.

Together with a very precise measurement of the $B_{s}^{0}$ mixing parameters and improved theoretical understanding of the model, the LHCb upgraded experiment could arguably exclude completely, or confirm, B-mesogenesis as the under- 
lying mechanism for the baryon asymmetry of the universe as well as the explanation for the Dark Matter problem.

Acknowledgements We would like to thank Miguel Escudero, Gonzalo Alonso-Álvarez, Gilly Elor, Luca Silvestrini, Kristof De Bruyn, Lucia Grillo, and Alex Lenz for useful discussions. This work has received financial support from Xunta de Galicia (Centro singular de investigación de Galicia accreditation 2019-2022), by European Union ERDF, and by the "María de Maeztu" Units of Excellence program MDM-2016-0692 and the Spanish Research State Agency. In particular: the work of X.C.V. is supported by MINECO (Spain) through the Ramón y Cajal program RYC-2016-20073 and by XuntaGAL under the ED431F 2018/01 project.

Data Availability Statement This manuscript has no associated data or the data will not be deposited. [Authors' comment: This sensitivity study is based on Pythia simulations, which should be easy to reproduce.]

Open Access This article is licensed under a Creative Commons Attribution 4.0 International License, which permits use, sharing, adaptation, distribution and reproduction in any medium or format, as long as you give appropriate credit to the original author(s) and the source, provide a link to the Creative Commons licence, and indicate if changes were made. The images or other third party material in this article are included in the article's Creative Commons licence, unless indicated otherwise in a credit line to the material. If material is not included in the article's Creative Commons licence and your intended use is not permitted by statutory regulation or exceeds the permitted use, you will need to obtain permission directly from the copyright holder. To view a copy of this licence, visit http://creativecomm ons.org/licenses/by/4.0/.

Funded by SCOAP ${ }^{3}$.

\section{Appendix A: Effect of systematic uncertainties}

The sensitivity results presented in this document make no assumptions on the systematic uncertainties. However, as mentioned in Sect. 5, one would expect them to limit the actual experimental analysis. We expect the dominant systematic uncertainty to arise from the knowledge of the actual background yield, which originates, among others, from the uncertainty in the $b \bar{b}$ cross section [15]. While any experimental analysis will do its best to cure this uncertainty, this will eventually limit the precision to be achieved. Potential strategies to mitigate this uncertainty include the use of heavier $b$ resonances to tag the events of interest, as discussed in Sect. 3.5, the introduction of smarter selection criteria to discriminate the signal against the background or the use of control regions that would provide a handle to determine the actual background content in the signal region.

To provide a more quantitative estimate of the effect of the systematic uncertainty in the background yield, we repeat the results of Sect. 4 for two of our benchmark channels, with the same methodology but now under different assumptions on the value of this uncertainty. These can found in Figs. 6 and 7 for the $B^{0} \rightarrow \psi_{\mathrm{DS}} \Lambda(1520)$ and $B^{+} \rightarrow \psi_{\mathrm{DS}} \Lambda_{c}(2595)^{+}$ channels, respectively. The main conclusion is that, in a
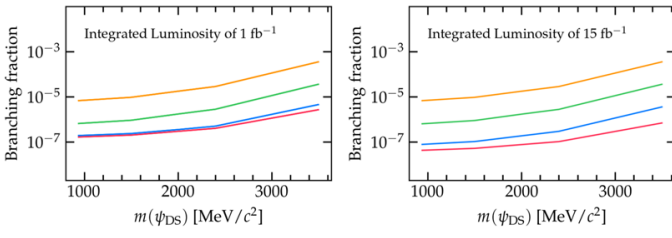

Systematics
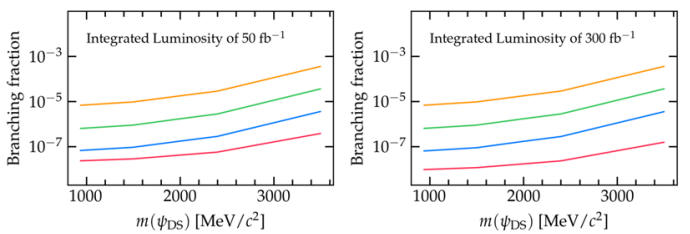

$-1 \%$

$10 \%$

No

Fig. 6 Projected sensitivity in terms of branching fractions excluded at $95 \% \mathrm{CL}$ for the $B^{0} \rightarrow \psi_{\mathrm{DS}} \Lambda(1520)$ channel. The sensitivity is shown assuming integrated luminosities of $1,15,50$ and $300 \mathrm{fb}^{-1}$. In each case, different assumptions are made regarding the systematic uncertainty of the background yield, ranging from no uncertainty at all to $10 \%$
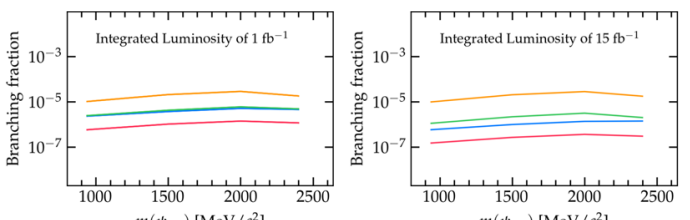

Systematics $m\left(\psi_{\mathrm{DS}}\right)\left[\mathrm{MeV} / c^{2}\right]$
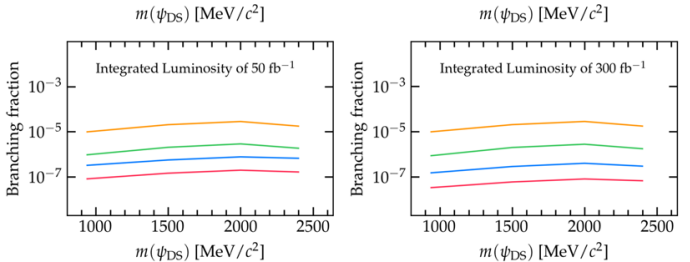

$-1 \%$
$-1 \%$

$10 \%$

- No

Fig. 7 Same as Fig. 6, but now for the $B^{+} \rightarrow \psi_{\mathrm{DS}} \Lambda_{c}(2595)^{+}$channel

pessimistic scenario, a systematic uncertainty of $O(10) \%$ would make the search systematically limited already with $\sim 1 \mathrm{fb}^{-1}$, compromising the reach of all the region of theoretical interest, with branching fractions $\gtrsim 10^{-5}$, for the channels of interest and any value of $m_{\psi_{\mathrm{DS}}}$. This region would be completely at reach with uncertainties of $O(1) \%$ or smaller. Finally, bringing the uncertainty down to the level per-mile would increase the systematic limit of the search to $\sim 15 \mathrm{fb}^{-1}$.

\section{References}

1. G. Elor, M. Escudero, A. Nelson, Phys. Rev. D 99(3), 035031 (2019). https://doi.org/10.1103/PhysRevD.99.035031

2. G. Alonso-Álvarez, G. Elor, M. Escudero, Phys. Rev. D 104(3), 035028 (2021). https://doi.org/10.1103/PhysRevD.104.035028

3. Y.S. Amhis et al., Eur. Phys. J. C 81(3), 226 (2021). https://doi.org/ 10.1140/epjc/s10052-020-8156-7. arXiv:1909.12524. Updated results and plots available at https://hflav.web.cern.ch

4. A. Lenz, G. Tetlalmatzi-Xolocotzi, JHEP 07, 177 (2020). https:// doi.org/10.1007/JHEP07(2020)177 
5. H.M. Asatrian, H.H. Asatryan, A. Hovhannisyan, U. Nierste, S. Tumasyan, A. Yeghiazaryan, Phys. Rev. D 102(3), 033007 (2020). https://doi.org/10.1103/PhysRevD.102.033007

6. R. Barate et al., Eur. Phys. J. C 19, 213 (2001). https://doi.org/10. $1007 / \mathrm{s} 100520100612$

7. G. Elor, R. McGehee, Phys. Rev. D 103(3), 035005 (2021). https:// doi.org/10.1103/PhysRevD.103.035005

8. F. Elahi, G. Elor, R. McGehee, report name: MITP-21-041 (2021). arXiv:2109.09751

9. V. Chobanova, D. Martínez Santos, C. Prouve, M. Romero Lamas (2020). arXiv:2012.02692

10. M. Borsato et al. (2021). arXiv:2105.12668

11. I. Bediaga et al. (2013). CERN-LHCC-2013-021. https://cds.cern. $\mathrm{ch} / \mathrm{record} / 1624070$

12. I. Bediaga et al. (2012). CERN-LHCC-2012-007. https://cds.cern. $\mathrm{ch} / \mathrm{record} / 1443882$

13. R. Aaij et al., Technical report (2018). https://cds.cern.ch/record/ 2636441. ISBN:978-92-9083-494-6

14. T. Sjöstrand, S. Ask, J.R. Christiansen, R. Corke, N. Desai, P. Ilten, S. Mrenna, S. Prestel, C.O. Rasmussen, P.Z. Skands, Comput. Phys. Commun. 191, 159 (2015). https://doi.org/10.1016/j.cpc.2015.01. 024

15. R. Aaij et al., Phys. Rev. Lett. 118(5) (2017). https://doi.org/10. 1103/PhysRevLett.118.052002 [Erratum: Phys. Rev. Lett. 119(16), 169901 (2017). https://doi.org/10.1103/PhysRevLett.119.169901]

16. R. Aaij et al., Phys. Rev. D 104(3), 032005 (2021). https://doi.org/ 10.1103/PhysRevD.104.032005

17. R. Aaij et al., Technical report, CERN, Geneva (2017). https://cds. cern.ch/record/2244311

18. A.A. Alves Jr. et al., CERN-LHCC-2014-001 (2014). https://cds. cern.ch/record/1647400

19. X. Cid Vidal, P. Ilten, J. Plews, B. Shuve, Y. Soreq, Phys. Rev. D 100(5), 053003 (2019). https://doi.org/10.1103/PhysRevD.100. 053003
20. X. Cid Vidal, Y. Tsai, J. Zurita, JHEP 01, 115 (2020). https://doi. org/10.1007/JHEP01(2020)115

21. P.A. Zyla et al., PTEP 2020(8), 083 C01 (2020). https://doi.org/10. 1093/ptep/ptaa104

22. R. Aaij et al., Phys. Lett. B 699, 330 (2011). https://doi.org/10. 1016/j.physletb.2011.04.031

23. D. Karlen, Comput. Phys. 12(4), 380 (1998). https://doi. org/10.1063/1.168691. https://aip.scitation.org/doi/abs/10.1063/ 1.168691

24. I. Bediaga et al., Technical Report. CERN-LHCC-2013-022. LHCB-TDR-014 (2013). https://cds.cern.ch/record/1624074

25. M. Adinolfi et al., Eur. Phys. J. C 73, 2431 (2013). https://doi.org/ 10.1140/epjc/s10052-013-2431-9

26. S. Stone, L. Zhang, Adv. High Energy Phys. 2014, 931257 (2014). https://doi.org/10.1155/2014/931257

27. R. Aaij et al., Phys. Rev. Lett. 122(1), 012001 (2019). https://doi. org/10.1103/PhysRevLett.122.012001

28. R. Aaij et al., JHEP 06, 129 (2020). https://doi.org/10.1007/ JHEP06(2020)129

29. T. Junk, Nucl. Instrum. Methods A 434, 435 (1999). https://doi. org/10.1016/S0168-9002(99)00498-2

30. A.L. Read, J. Phys. G 28, 2693 (2002). https://doi.org/10.1088/ 0954-3899/28/10/313 